\title{
Fabrication Procedure for Rugged and Breathable Forms of Stretchable Electronics with Adherent and Composite Substrates
}

\author{
John A. Rogers ( $\sim$ jrogers@illinois.edu )
}

Rogers Research Group, University of Illinois at Urbana-Champaign

Huanyu Cheng

Department of Civil and Environmental Engineering, Department of Mechanical Engineering, Center for Engineering and Health and Skin Disease Research Center, Northwestern University

\section{Gabriele Gratton}

Department of Psychology, University of Illinois at Urbana-Champaign

\section{Monica Fabiani}

Department of Psychology, University of Illinois at Urbana-Champaign

\section{Ronald Carbonari}

Beckman Institute for Advanced Science and Technology, University of Illinois at Urbana-Champaign

John G. Gaspar

Beckman Institute for Advanced Science and Technology, University of Illinois at Urbana-Champaign

\section{Moongee Cho}

Rogers Research Group, University of Illinois at Urbana-Champaign

\section{Yiyuan Yang}

Rogers Research Group, University of Illinois at Urbana-Champaign

\section{Dongjun Lee}

Rogers Research Group, University of Illinois at Urbana-Champaign Ha Uk Chung

Rogers Research Group, University of Illinois at Urbana-Champaign

\section{Byunggik Kim}

Rogers Research Group, University of Illinois at Urbana-Champaign

\section{Jeahoon Chung}

Rogers Research Group, University of Illinois at Urbana-Champaign

\section{Sang II Rhee}

Rogers Research Group, University of Illinois at Urbana-Champaign

\section{Stanley Kim}

Rogers Research Group, University of Illinois at Urbana-Champaign

\section{Kyung-In Jang}

Rogers Research Group, University of Illinois at Urbana-Champaign 


\section{Woon-Hong Yeo}

Department of Mechanical and Nuclear Engineering, Virginia Commonwealth University

\section{Young Min Song}

Department of Electronic Engineering, Pusan National University

\section{Rak Hwan Kim}

Rogers Research Group, University of Illinois at Urbana-Champaign

Thomas J. Dawidczyk

Rogers Research Group, University of Illinois at Urbana-Champaign

\section{Jung Woo Lee}

Rogers Research Group, University of Illinois at Urbana-Champaign

\section{R. Chad Webb}

Rogers Research Group, University of Illinois at Urbana-Champaign

\section{Gwang-Tae Kim}

Rogers Research Group, University of Illinois at Urbana-Champaign

\section{Jae-Woong Jeong}

Rogers Research Group, University of Illinois at Urbana-Champaign

\section{Yihui Zhang}

Department of Civil and Environmental Engineering, Department of Mechanical Engineering, Center for Engineering and Health and Skin Disease Research Center, Northwestern University

\section{Kyle E. Mathewson}

Department of Psychology, University of Alberta

\section{Sheng Xu}

Rogers Research Group, University of Illinois at Urbana-Champaign

\section{Sang Youn Han}

Samsung Display Co. Display R\&D Center

\section{Yonggang Huang}

Department of Civil and Environmental Engineering, Department of Mechanical Engineering, Center for Engineering and Health and Skin Disease Research Center, Northwestern University

\section{Method Article}

Keywords: Stretchable electronics, Wearable electronics, Smart textile

Posted Date: July 10th, 2014

DOI: https://doi.org/10.1038/protex.2014.020

License: (9) This work is licensed under a Creative Commons Attribution 4.0 International License. Read Full License 


\section{Abstract}

We introduce materials and composite designs for thin, breathable, soft electronics that can adhere strongly to the skin. In this protocol, we describe step-by-step procedure of device preparation with helpful information.

\section{Procedure}

Step 1: Preparation of substrate for temporary use in device mounting Timing: 2 hours 1. Prepare a glass slide with the dimension of $75 \mathrm{~mm} \times 50 \mathrm{~mm} \times 1.0 \mathrm{~mm}$. 2. Spin cast poly $\backslash$ (methylmethacrylate) $\backslash(P M M A)$ at $3000 \mathrm{rpm}$ for 30 seconds and cure on a hot plate at $180^{\circ} \mathrm{C}$ for 3 minutes. 3 . Spin cast polyimide at 3000 rpm for 30 seconds and cure inside a vacuum oven at $250^{\circ} \mathrm{C}$ for 1.5 hours. Step 2: Fabrication of wireless heater Timing: 2 hours 4. Deposit metals $\backslash(\mathrm{Cr} / \mathrm{Au}, 15 / 100 \mathrm{~nm})$ on the prepared substrate using electron beam evaporator. 5. Define open mesh architecture composed of narrow filament serpentine $\backslash(\mathrm{FS})$ traces by photolithography $\backslash$ (positive photoresist, AZ 5214). Perform wet chemical etching using $\mathrm{Cr}$ and $\mathrm{Au}$ etchant and remove photoresist with acetone Step 3: Fabrication of RF antenna Timing: 4 hours 6. Deposit copper $\backslash(\mathrm{Cu}, 3 \mu \mathrm{m})$ on the substrate using electron beam evaporation. 7. Define dipole geometry with FS mesh layout by photolithography \(positive photoresist, AZ 5214). Perform wet chemical etching using $\mathrm{Cu}$ etchant and remove photoresist with acetone. 8. Spin cast polyimide on glass slide at $3000 \mathrm{rpm}$ for 30 seconds and cure inside a vacuum oven at $250^{\circ} \mathrm{C}$ for 1.5 hours. Step 4: Fabrication of $\mu$-ILED Timing: 2 days 9. Prepare AllnGaP epitaxial layer stacks by MOCVD growth on a GaAs wafer $\backslash(670 \mathrm{~nm}$ emission wavelength; $\mathrm{p}$-spreading layer $\backslash(\mathrm{Al0}$.45Ga0.55As:C)/p-cladding layer $\backslash$ (In0.5AI0.5P:Zn)/quantum well \(Al0.25Ga0.25In0.5P/In0.56Ga0.44P/Al0.25Ga0.25In0.5P)/ n-cladding \ (In0.5Al0.5P:Si)/n-spreading layer $\backslash($ Al0.45Ga0.55As:Si)/n-GaAs:Si/sacrificial layer \ (Al0.96Ga0.04As)/undoped GaAs). 10. Deposit silicon dioxide $\backslash(600 \mathrm{~nm})$ on the wafer using PECVD. Define arrays of L-shaped patterns $\backslash(280 \mu \mathrm{m} \times 280 \mu \mathrm{m})$ by photolithography $\backslash$ (positive photoresist, AZ 5214). 11. Immerse the wafer in buffered oxide etch $\backslash(6: 1 \mathrm{NH} 4 \mathrm{~F}: \mathrm{HF})$ for 3 minutes at ambient temperature. $\triangle$ CRITICAL STEP Dimension of $\mu$-ILED is determined in this step. Under etch results in failure during ICP RIE process. 12. Rinse the wafer in a tub of DI water for 10 seconds then transfer to final rinse tub for 20 seconds. 13. Immerse the wafer in diluted hydrochloric acid solution $\backslash(\mathrm{DI}$ water: $\mathrm{HCl}$ $=1: 1$ ) for 15 seconds. Rinse the wafer in DI water then remove photoresist with acetone. 14. Open $\mathrm{n}$ contact using ICP RIE $\backslash\left(25^{\circ} \mathrm{C}, 2 \mathrm{mTorr}, 6 \mathrm{sccm} \mathrm{Cl} 2,3 \mathrm{sccm} \mathrm{H} 2,6 \mathrm{sccm}\right.$ Ar, 5 minutes $)$. Measure height profile with profilometer to confirm that $\mathrm{n}$-contact is opened. 15. Define arrays of square patterns $\backslash(300 \mu \mathrm{m} \times 300$ $\mu \mathrm{m})$ to physically distinguish $\mu$-ILEDs by photolithography $\backslash$ (positive photoresist, SPR 220 7.0). Immerse the wafer in n-contact etch solution \(H3PO4: DI water: H2O2 = 1: 12: 13) for 30 seconds. Rinse the wafer with DI water. 16. Immerse the wafer in diluted hydrofluoric acid $\backslash(D I$ water: HF $=1: 10)$ for 3 seconds to enhance subsequent undercut etching process for sacrificial layers. Remove photoresist with acetone. 17. Define n-contact pads $\backslash(80 \mu \mathrm{m} \times 80 \mu \mathrm{m})$ by photolithography $\backslash(A Z 5214)$. Immerse the wafer in diluted hydrochloric acid $\backslash(\mathrm{DI}$ water: $\mathrm{HCl}=1: 1)$ for 15 seconds and rinse with DI water. 18. Deposit n-contact metals $\backslash(\mathrm{Pd} / \mathrm{Ge} / \mathrm{Au}, 5 / 35 / 70 \mathrm{~nm})$ using electron beam evaporation and lift-off photoresist by immersing 
the wafer in acetone. 19. Define p-contact pad $\backslash(80 \mu \mathrm{m} \times 80 \mu \mathrm{m})$ by photolithography $\backslash(P R 5214)$. Immerse the wafer in buffered oxide etch $\backslash(6: 1 \mathrm{NH} 4 \mathrm{~F}: \mathrm{HF})$ for 3 minutes to open $\mathrm{p}$-contact pad and rinse with water. 20. Deposit p-contact metals $\backslash(\mathrm{Pt} / \mathrm{Ti} / \mathrm{Pt} / \mathrm{Au}, 10 / 40 / 40 / 50 \mathrm{~nm})$ using electron beam evaporation and lift-off photoresist. 21. Anneal the wafer at $300^{\circ} \mathrm{C}$ for 30 minutes inside a glove box to generate Ohmic contacts. 22. Define anchoring pattern to enable transfer mechanism by photolithography $\backslash\left(\right.$ SPR 2207.0 ) and hard bake photoresist at $130^{\circ} \mathrm{C}$ for 10 minutes. 23. Immerse the wafer in diluted hydrofluoric acid $\backslash(D I$ water: $H F=1: 20)$ for 2 hours to undercut the sacrificial layer. Transfer wafer to tub of DI water for initial rinse for 1 minute and final rinse for 3 minutes. $\triangle$ CRITICAL STEP The arrays of $\mu$-ILED may float during rinsing. Softly transfer wafer to DI water tub and do not swirl the water. Step 5: Preparation of polydimethylsiloxane \(PDMS) stamp for transfer printing Timing: 1 day 24. Prepare a glass slide $\backslash(75 \mathrm{~mm} \times 50 \mathrm{~mm} \times 1.0 \mathrm{~mm})$. 25. Define a pattern of squares $\backslash(320 \mu \mathrm{m} \times 320$ $\mu \mathrm{m})$ by photolithography $\backslash$ (epoxy negative photoresist, SU-8 2050). Cure SU-8 at $150^{\circ} \mathrm{C}$ for 30 minutes. 26. Silanize using tridecafluoro-1,1,2,2-tetrahydrooctyl trichlorosilane $\backslash($ UCT Specialties, LLC). 27. Deposit PDMS $\backslash(10: 1)$ on the prepared surface and cure for 1 day at ambient temperature. Step 6: Transfer printing $\mu$-ILED on the glass substrate Timing: 2 days 28. Define alignment marks on the prepared glass substrate $\backslash$ (wireless heater system fabricated) for transfer printing by photolithography $\backslash(A Z$ 5214). 29. Deposit metal $\backslash(\mathrm{Cu}, 200 \mathrm{~nm})$ using electron beam evaporation and lift-off photoresist. 30 . Retrieve the $\mu-$ ILED \(s) using a PDMS stamp prepared using a 1:10 mixture of base to curing agent $\backslash$ (Sylgard 184, Dow Corning). 31. Spin cast polyimide at $3000 \mathrm{rpm}$ for 30 seconds on the substrate to serve as an adhesion layer. $\triangle$ CAUTION A few minutes after spin casting, polyimide starts curing at ambient temperature and loses its tacky, adhesive surface. Transfer printing of $\mu$-ILEDs should be performed before this loss of adhesion. 32. Cure polyimide partially at $130^{\circ} \mathrm{C}$ for 10 minutes then remove photoresist anchor structures that remain on the $\mu$-ILED by spraying acetone. Cure polyimide fully inside a vacuum oven at $250^{\circ} \mathrm{C}$ for 1.5 hours. 33. Define $n$-type and p-type contact pads of the $\mu$-ILEDs for metal interconnection by photolithography $\backslash\left(\right.$ SU-8 2002). Cure SU-8 at $150^{\circ} \mathrm{C}$ for 30 minutes. Step 7: Fabrication of electrophysiological sensor and metal interconnection Timing: 1 day 34. Deposit metals $\backslash(\mathrm{Cr} / \mathrm{Au}, 5 / 60$ $\mathrm{nm}$ ) using a sputtering system. $\triangle$ CRITICAL STEP Sputtering provides sidewall deposition that is critical for $\mu$-ILED metal interconnection. 35. Define electrophysiological sensor and metal connect pattern for wireless heater system and $\mu$-ILED by photolithography $\backslash(A Z$ 5214). Perform wet chemical etching using $\mathrm{Au}$ and $\mathrm{Cr}$ etchants then remove photoresist. 36. Spin cast polyimide at $3000 \mathrm{rpm}$ for 30 seconds and cure inside a vacuum oven at $250^{\circ} \mathrm{C}$ for 1.5 hours. 37. Deposit copper $\backslash(\mathrm{Cu}, 20 \mathrm{~nm})$ using electron beam evaporation. 38. Define regions where polyimide should be etched away by photolithography $\backslash(A Z 5214)$. Perform wet chemical etching using $\mathrm{Cu}$ etchant then remove photoresist. 39. Etch polyimide by reactive ion etching $\backslash$ (March RIE, 300mTorr, 200W, 20sccm 02, 20 mintues). Remove copper using Cu etchant then degrease the fabricated device by immersion in acetone, isopropyl alcohol, and DI water. Step 8: Preparation of textile substrate and transfer printing fabricated system Timing: 1 day 40. Prepare textile substrate. Spin cast Silbione at $1000 \mathrm{rpm}$ for 60 seconds then cure at room temperature for 1 day. 41 . Stack another glass slide $\backslash(75 \mathrm{~mm} \times 50 \mathrm{~mm} \times 1.0 \mathrm{~mm}) /$ cleanroom wipe on the fabricated system and hold them with a binder clip. Next, immerse in acetone to remove PMMA. $\triangle$ CRITICAL STEP Fabricated devices may float around inside the acetone solution without glass slide/cleanroom wipe. 42. Dry sample and 
remove glass slide/cleanroom wipe. Attach water soluble tape on the surface of fabricated system. Retrieve the system by slowly removing the tape from the glass slide. 43. Attach the tape on the Silbione/textile substrate then immerse in water to remove the tape.

\section{Figures}

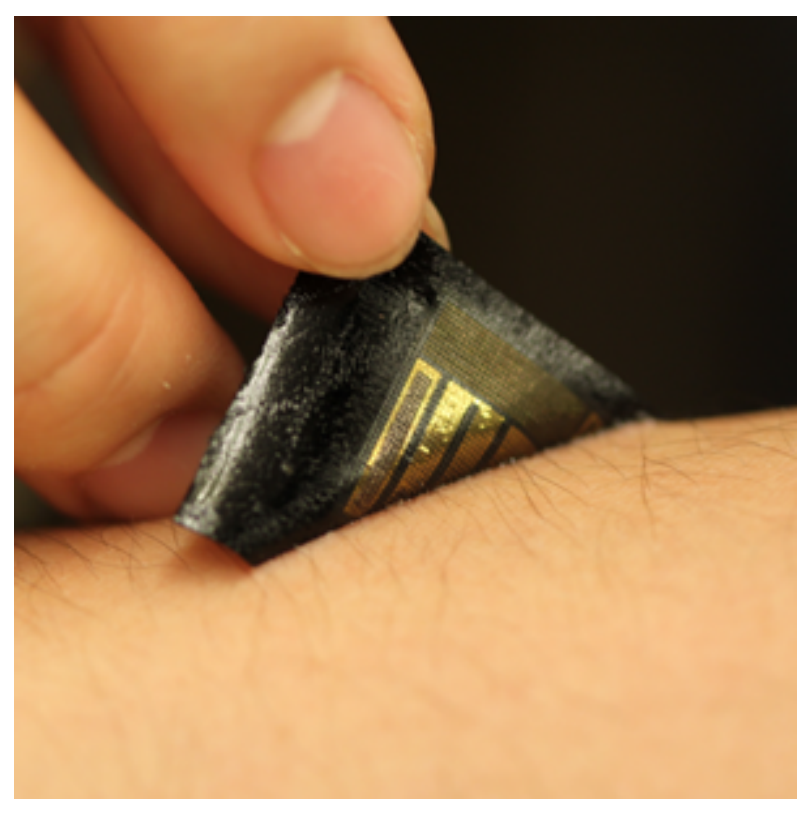

\section{Figure 1}

An attached device onto the skin

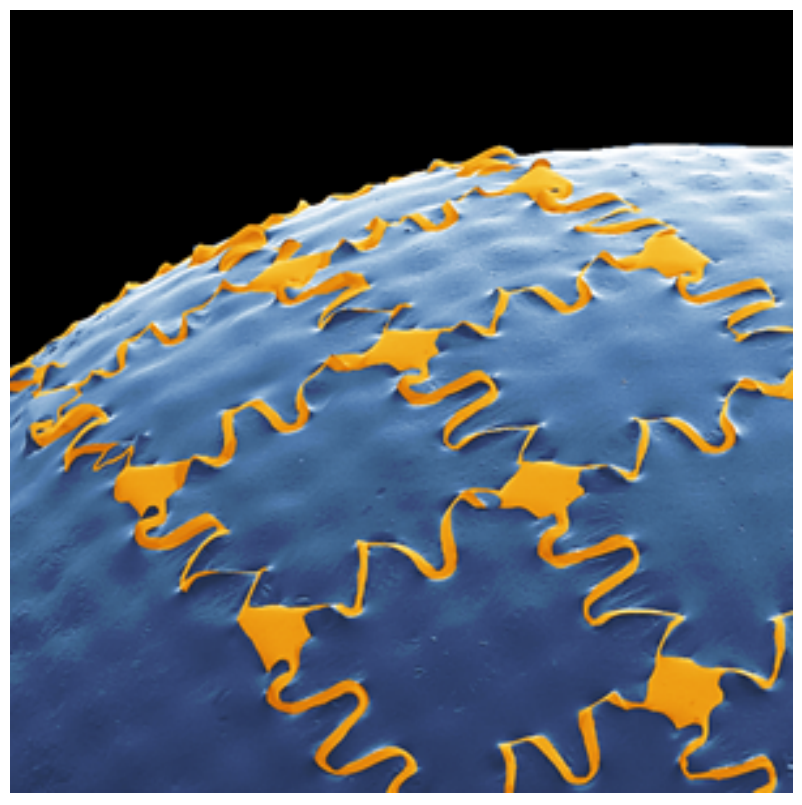

Figure 2

A SEM image of a biaxially stretched device 